\title{
IOT BASED HEALTHCARE SYSTEM FOR SIGNAL MONITORING USING CLOUD TECHNOLOGY
}

\author{
Sonal R. Chakole \\ Assistant Professor \\ Priyadarshini J. L. College of Engg. \\ Nagpur, India \\ Dr. V.G. Giripunje \\ Assistant Professor \\ Priyadarshini College of Engg . \\ Nagpur, India
}

\author{
Bharat Dhak \\ Assistant Professor \\ Priyadarshini J. L. College of Engg \\ Nagpur, India \\ Mausami Sawarkar \\ Assistant Professor \\ Priyadarshini J. L. College of Engg. \\ Nagpur, India
}

\begin{abstract}
To provide economic telemecine to the patients living in the interior part of the globe is the need today. So as to serve such patients efficiently, a low power, low cost, highly portable wearable wireless telemedicine system may be a possible solution. In this paper, a cloud-based physiological signal monitoring system implemented for continuous cardiac monitoring is presented. The system consists of an ECG sensor Module, one wire digital temperature sensor, an ultra low power MSP432 MCU and IOT Module. The system presented in this paper acquires body temperature by one wire digital thermometer DS18B20 without any extra ADC hardware. A power efficient 3-lead ECG sensor module AD8232 captures ECG and hence measures heart rate. The temperature value and ECG signal acquired from respective sensors are processed by an ultra low power high speed cortex core MSP432P401R processor/controller. In This paper it is tried to transmit important as well as an essential physiological signals of the patient over the globe using cloud technology. The temperature and the ECG signals are transmitted on to the cloud with the help of ESP 8266 Node MCU. The signals at transmitting and receiving end are compared and analyzed. This system proposed in this paper seems to be an efficient solution for wireless patient monitoring using cloud technology which is distributed data processing in which some scalable information resources and capacities are provided as a service to multiple external customers through Internet technology.
\end{abstract}

Keywords-telemetry; EC Gsensor; healthcare; Instrumentation; MSP432processor; node $\mathrm{MCU}$; IOT Internet of things

\section{INTRODUCTION}

Healthcare applications utilizing body sensor networks generate a vast amount of data that need to be managed and stored for processing and future usage. Cloud computing among with the Internet of Things (IoT) concept is a new trend for efficient managing and processing of sensor data online. This paper presents a platform based on Cloud Computing for management of mobile and wearable healthcare sensors, demonstrating this way the IoT paradigm applied on pervasive healthcare. The Internet of Things (IoT) connects set of anyone, anything, anytime, anyplace, any service, and any network. IOT is used to globalize the patient data. IOT is useful in many applications such as health care, water level indicator, security, HMIS (Hospital management integrates service) etc. In this paper, monitoring of biological parameters such as body temperature and ECG/heart rate are acquired using low power sensors. These signals are properly processed by an ultra low power MSP432 processor before being fed to UART pin for the transmission to Node MCU. In the IOT based patient monitoring system, the real-time parameters of patient's health are sent to cloud using Internet for connectivity. $[1,5]$

The patient's physiological information is sent to a remote Internet location so that an examiner/doctor can view these details from anywhere in the world. Doctors can take the reference of these changes or the history of the patient while suggesting the treatment or the medicines to the patient.

Patient's real time health status can be stored in the cloud. So it is more convenient to the doctors rather than maintaining the records on printed papers kept in the files or even the digital records which are kept in a particular computer or laptop or memory device like a pen- drive. Because there are chances that these devices can get corrupt and data might be lost. 
Whereas in case of IOT, the cloud storage is more reliable with minimum chances of data loss. [2, 3, 4]

\section{SYSTEM BLOCK DIAGRAM}

The system block diagram shown in fig. 1. consists of a temperature sensor, heart rate and ECG sensor, MSP432, Power supply, IOT module ESP 8266 (node MCU) and the receiving section i.e. mobile or laptop. A temperature sensor (1 Wire digital Thermometer) will detect human body temperature and then the data will be given to MSP432 by converting the signal from analog to digital.

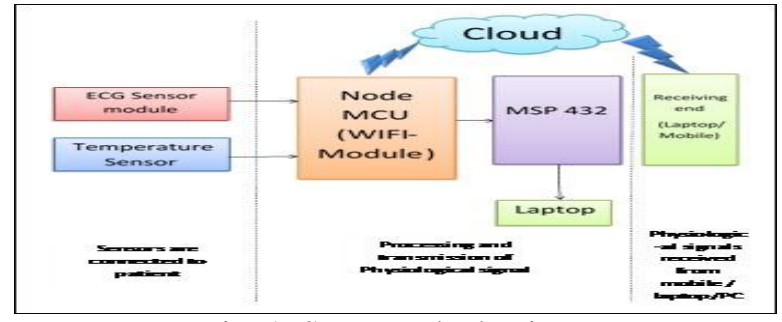

Fig. 1. System Block Diagram

In this paper the health care system is implemented to monitor physiological parameters of the patient using internet of things (IoT) and to improve the system performance with respect to power consumption, speed, and cost of the system. The block diagram consists of ECG sensor module, temperature sensor, MSP432 microcontroller and $\mathrm{Wi}-\mathrm{Fi}$ module at the transmitting end and Laptop/PC/Mobile at the receiving/examiner end. The one wire digital thermometer DS18B20 takes body temperature directly in the digital form so is fed directly to the processor unit. The AD8232 is an integrated ECG signal conditioning unit with 3-leads arrangements and is used to acquire an ECG/Heart rate. The small voltage ECG signals in analog form are converted into digital form with the help of an inbuilt ADC. The converted digital ECG signal is fed to MSP432 MCU. MSP432 after due processing the temperature and ECG/heart rate signals puts them on to the UART pin for their transmission to the Node MCU. These signals are then transmitted to the cloud where they are stored as data base of the patient. The receiver (doctor/examiner) can access this data from the cloud by using Wi-Fi, internet etc. [6, 7]

There are the different ranges of body temperature which varies according to their ages are given in table I
TABLE I. STANDARD BODY TEMPERATURE RANGES OF PATIENT

\begin{tabular}{|c|c|c|}
\hline \multicolumn{3}{|c|}{ Normal Body Temperature } \\
\hline $\begin{array}{l}\text { Age in } \\
\text { Years }\end{array}$ & Temp. in ${ }^{\mathbf{0}} \mathbf{C}$ & Temp. in ${ }^{\mathbf{}} \mathbf{F}$ \\
\hline $0-2$ & $36.4-38.1$ & $97.5-100.4$ \\
\hline $3-10$ & $36.1-37.8$ & $97.0-100.0$ \\
\hline $11-65$ & $35.9-37.6$ & $96.6-99.7$ \\
\hline$>65$ & $35.8-37.5$ & $96.4-99.5$ \\
\hline
\end{tabular}

\section{HARDWARE DESCRIPTION}

\section{A. MSP 432P-401R as a MCU in the System}

MSP432P401-R MCU (32 bit processor) provides freedom to the developer to maximize the performance of the system with low power budget as it do not consumes more than $95 \mu \mathrm{A} / \mathrm{MHz}$ in active mode and $850 \mathrm{nA}$ in standby mode (including RTC). Therefore it is useful to design an ultra-low- power embedded systems where leakage current has no significant contribution and hence helps to increase battery life of wearable health monitoring unit. MSP432P401-R has an ultra-low-power mode (LPM3) which draws standby current as minimum as $850 \mathrm{nA}$. Additively it has many features with which flexibility in hardware design as well as software development is provided and therefore it is best suited for biomedical applications. Hence due to ARM Cortex-M4F core the performance of MSP432P401-R processor has been at its best with an ultra-low power consumption benefit. $[8,9,10]$

\section{B. Sensor Modules}

The DS18B20 is the digital sensor for body temperature measurements and provides alarm function for exceeding temperature. It communicates with only single wire bus and so requires one data line only. It operates over the temperature range of $-55^{\circ} \mathrm{C}$ to $+125^{\circ} \mathrm{C}$ with resolution of $\pm 0.5^{\circ} \mathrm{C}$ In addition, the DS18B20 sinks power from the data line itself and therefore an external power supply remains optional. It is therefore can be best suited sensor for body temperature telemetry systems. [11]

The AD8232 is an integrated signal conditioning unit for ECG signal and is especially designed to acquire, amplify, and filter very small bio potential signals in mili volts even in the noisy situations, such as those created by blood motion in the body or distant electrode placement. The module can be very efficient in fitness and activity heart rate monitors, portable ECG machines, remote health care monitors etc. The module is featured with only one lead in front end which draws just $170 \mu \mathrm{A}$ with $2 \mathrm{~V}$ to $3.5 \mathrm{~V}$ supply voltage. It has an inbuilt two pole adjustable high pass filter and three pole adjustable low pass filter which are required for the development of 
sensor hardware. Along with high CMRR ( $80 \mathrm{~dB}$ for 0 $\mathrm{Hz}$ to $60 \mathrm{~Hz}$ ) AD8232 module has the capability to acquire a very small voltage (up to $\pm 300 \mathrm{mV}$ ). Hence, even a small bio potential signals can be acquired with maximum accuracy. [12]

\section{Node MCU (ESP8266 Wi-Fi module)}

ESP8266 has been designed for mobile, wearable electronics and Internet of Things applications to consume least possible power. The power saving architecture operates in 3 modes: active mode, sleep mode and deep sleep mode. ESP8266 consumes less than 12uA in sleep mode and less than $1.0 \mathrm{~mW}$ or less than $0.5 \mathrm{~mW} \quad(\mathrm{DTIM}=10)$ to stay connected to the access point. In sleep mode, only the calibrated real-time clock and watchdog remains active. The real-time clock can be programmed to wake up the ESP8266/ESP-12E at any required interval. [12, 15, 16]

\section{SYSTEM IMPLEMENTATION}

\section{A. Sensors Interfacing with MSP432 MCU}

The interfacing of hardware is shown in above fig 2 . It consists of laptop/PC, Microcontroller (MSP432), Node MCU (ESP8266), ECG sensor module (AD8232) and digital temperature sensor (DS18B20). The ECG sensor module pin no. LO+ is connected to MSP432 (pin no.28) and LO- is connected to MSP432 (pin no.29). Temperature sensor`s DQ pin is connected to the pin no. D2 of Node MCU and supply pins of MSP432 are commonly given to each device or module in the system. The output pin of ECG sensor module (AD8232) is directly given to pin no. 30 of MSP432 as well as Node MCU`s A0 pin to send the real-time data directly to a cloud. As ECG sensor module is used for measuring or monitoring the ECG and Heart Rate of the human body and the temperature sensor is used to measure the body temperature of the human body Fig. Shows important values of Hardware Interfacing between node MCU with temperature sensor ,ECG sensor module connected to Laptop or any device compatible with system. Solutions to the problem of formulating and managing Internet-connected objects in cloud environments i.e. environments comprising "entities" and resources (such as sensors, actuators and smart devices) in cloud infrastructures are still in their infancy. This paper formulates design principles for IoT cloud environments and introduces a framework for the converging utilitydriven cloud-based computing models, with emerging Internet-of- Things (IoT) infrastructures and applications into the cloud. Following results from analyzing the stateof-the-art and efforts towards the convergence of cloud computing and IoT, this paper also presents the main building blocks of a framework, which emphasizes ondemand establishment of IoT services based on the automated formulation of societies of Internet-connected objects. The framework leverages well- known technologies.

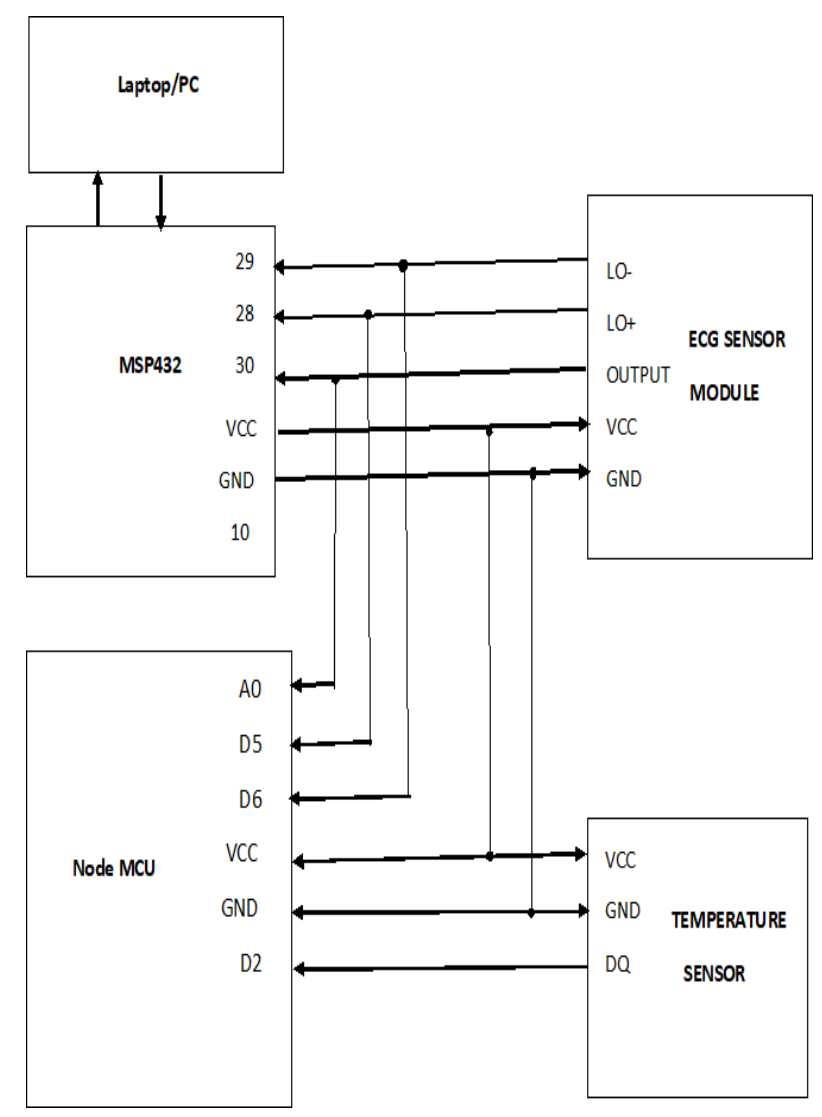

B. Implemented Healthcare System

The physiological monitoring system for ECG/heart rate and human body temperature monitoring using cloud technology is implemented and shown in fig. 3. The software logic developed for the system operation is also depicted in fig. 4.

\section{ADVANTAGES}

The staying of specialist is eliminated. It is multipurpose so that overall conditions are easily measured. Easy to operate.

Compare with compact sensors it gives better performance. Easy and reliable for doctors. Increases efficiency \& accuracy. 
Logic for software support to the Telemetry System
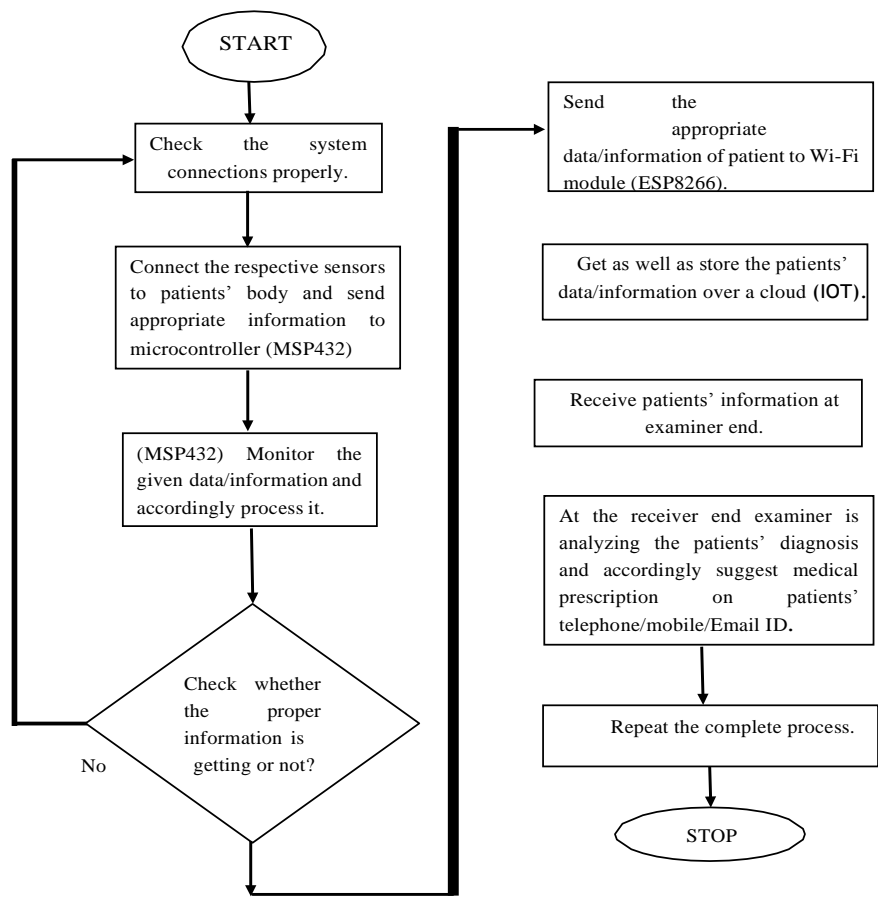

V.

RESULTS

A. ECG Signal wirelessly Obtained at Local PC

Even though the physiological signals are transmitted to the doctors/examiners for their analysis to diagnose the disease, the same signals can be made available to observe on the local at the patient's end also. The signal on local PC is shown in fig. 5 .

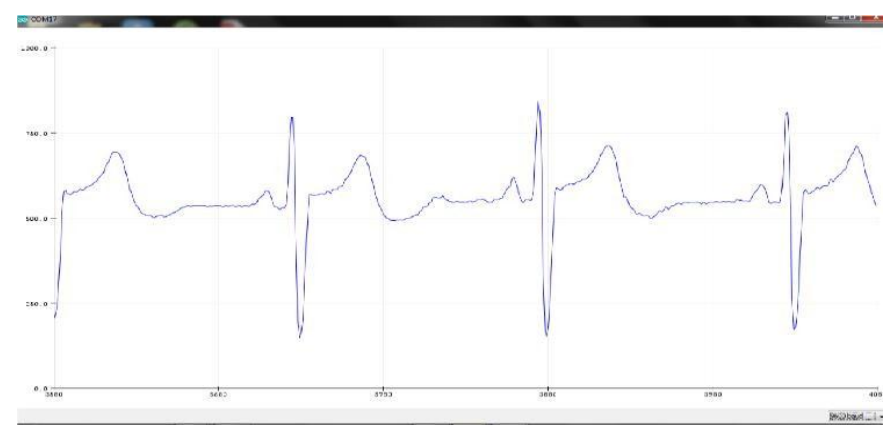

Fig. 5. ECG signal obtained at Local PC (Patient's End) ECG signal obtained follows the sequence of P Q R S T and is called as sinus rhythm. ECG \& Heart Rate Output at Node MCU \& Cloud ServerThe Heart rate signal and Temperature values of the patient and their time base variation at Node MCU server are obtained as shown in 6 and fig. 7 respectively whereas the ECG signal and temperature variations obtained at cloud server is shown in fig. 8 .

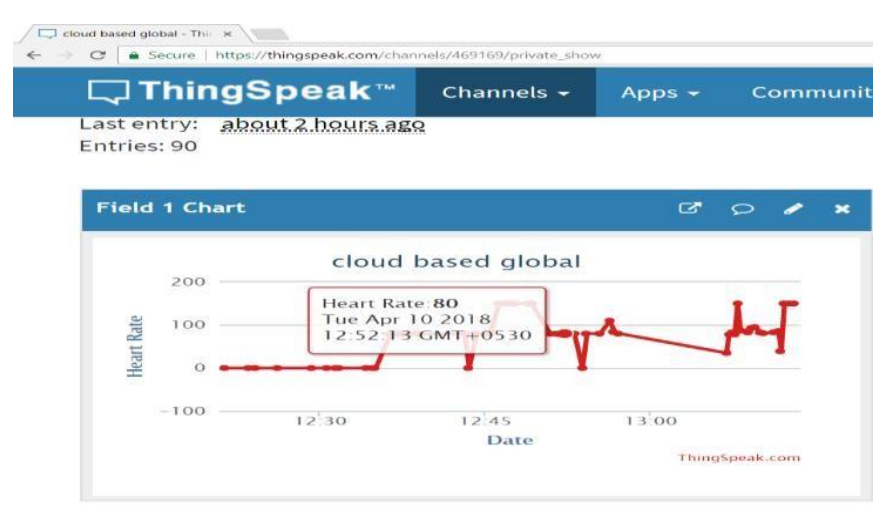

Fig. 6. Time base variation of heart rate at node MCU

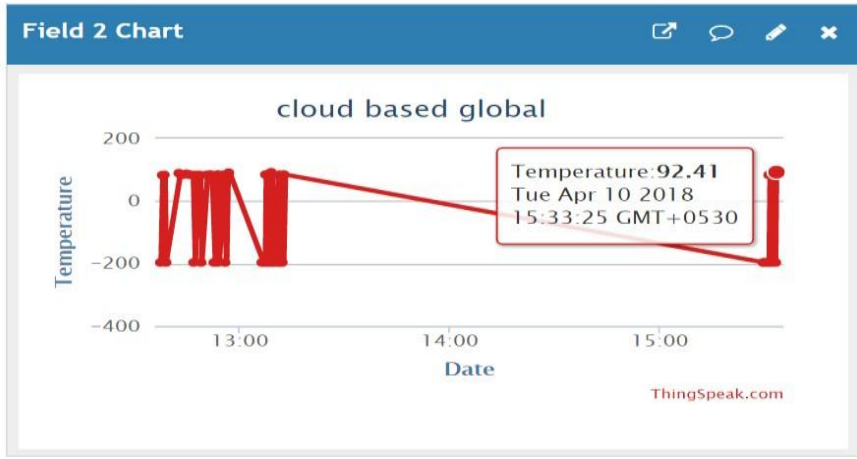

Fig. 7. Time base variation of Temperature at node MCU

Fig. 8. ECG signal and temperature variation obtained at cloud server. The signals/parameter values received are real time. The various observation of heart rate and temperature which are measured at different instant of time are tabulated in table II.

TABLE II. HEART RATE VARIATION WITH RESPECT TO TIME

\begin{tabular}{|c|c|c|c|}
\hline $\begin{array}{c}\text { Time } \\
\text { (HH:MM:SS) } \\
\text { AM/PM }\end{array}$ & $\begin{array}{c}\text { Heart } \\
\text { Rate }\end{array}$ & $\begin{array}{c}\text { Time } \\
\text { (HH:MM:SS) } \\
\text { AM/PM }\end{array}$ & Temperature \\
\hline 11:07:00 AM & 83 & $11: 07: 17 \mathrm{AM}$ & 83.3 \\
\hline 12:52:00 PM & 80 & $12: 52: 01 \mathrm{PM}$ & 84.09 \\
\hline 01:13:00 PM & 150 & $01: 13: 55 \mathrm{PM}$ & 84.65 \\
\hline 03:31:00 PM & 79 & $03: 31: 00 \mathrm{PM}$ & 96.6 \\
\hline 03:31:36 PM & 80 & $03: 31: 11 \mathrm{PM}$ & 83.53 \\
\hline 03:31:52 PM & 79 & $03: 31: 52 \mathrm{PM}$ & 83.64 \\
\hline $03: 33: 25 \mathrm{PM}$ & 80 & $03: 33: 00 \mathrm{PM}$ & 96.66 \\
\hline 03:33:57 PM & 79 & $03: 33: 25 \mathrm{PM}$ & 92.41 \\
\hline
\end{tabular}




\begin{tabular}{|c|c|c|c|}
\hline $03: 34: 00 \mathrm{PM}$ & 80 & $03: 34: 29 \mathrm{PM}$ & 87.24 \\
\hline $03: 34: 29 \mathrm{PM}$ & 82 & $03: 34: 44 \mathrm{PM}$ & 85.66 \\
\hline $03: 35: 50 \mathrm{PM}$ & 73 & $03: 35: 50 \mathrm{PM}$ & 84.2 \\
\hline $03: 36: 53 \mathrm{PM}$ & 80 & $03: 36: 37 \mathrm{PM}$ & 96.7 \\
\hline $03: 37: 41 \mathrm{PM}$ & 84 & $03: 37: 41 \mathrm{PM}$ & 83.64 \\
\hline
\end{tabular}

\section{CONCLUSION AND FUTURE SCOPE}

The components which are mentioned above are selected to achieve/develop high speed biomedical signal monitoring system. The processor and different sensor modules are selected on the basis of low power consumption, so as to develop low power, low cost, fastest system. The speed of the transmitting data is very high but the cloud server delay is $15 \mathrm{sec}$. So, sometimes there are possibilities of data loss. Due to this the ECG signal obtained at the output window of the cloud is bit distorted. But as the temperature is transmitted and received in terms of digit values the data loss situation do not occur.

However, Blood pressure (BP), Glucose, Brainwaves and other health parameters like real time EEG,EMG signals can also be monitored using same system. It is also possible to monitor more than a single patient at different places using such system. PHP, HTML, Java Script are three programming languages which are used to create one data base and with the help of that data base the ECG waveform can be obtained at the receiving end.

The human body scanning system could be made more sophisticated by incorporating blood pressure and EEG sensors. Hospitable-wide wireless capability would allow doctor to attend the patients' database using their word held computers. The entire medical data acquisitions could be made wireless and wearable. Such a package would contain the circuiting for inputs from ECG sensors, EEG sensors, pressure measurements and pulse rate transducers. This wearable module can transmit the data continuously over a fiber optic link or through an internet digital radio. The received data can be stored in separate memory and be processed by a microcontroller. This enhancement will enable monitoring of patients to be more flexible and strain free.

\section{REFERENCES}

[1] Udit Satija, Student Member, IEEE, Barathram.Ramkumar, and M. Sabarimalai Manikandan, Member, "Real-Time Signal QualityAware ECG Telemetry System for IoT-Based Health Care Monitoring" DOI 10.1109/JIOT.2017.2670022, IEEE.

[2] Seyedparham Mohajerani, Syed Ali Hashim Moosavi,
Rami-Al Rihawi, Beena Ahmed Texas A\&M University at Qatar Doha, Qatar "A Cloud- Based System for RealTime, Remote Physiological Monitoring of Infants" 2015 IEEE International Symposium on Signal Processing and Information Technology (ISSPIT).

[3] V.G.Girhepunje, Santosh Chede," Realization Development of Low Power Cardiac Telemetry System"2015 IEEE International Conference on Communication Networks ( November21, 2015, (Gwalior M.P,India)

ISBN: 978-1-5090-0051-7/15/\$31.00@2015 IEEE

[4] V.G.Girhepunje, SantoshChede," Realization of Low Power Telemedicine System to MonitorTemperature and Heart Beats"ICESA,October,30-November1, 2015,Pune(India) ISBN: 978-1- 4673-68179/15/\$31.00@2015 IEEE.Marci Meingast, Tanay Roosta, Shankar Sastry ,"Security \& Privacy issues with health care information technology", IEEE 2014

[5] V.Ramya, Anuradha Kumari, "Embedded Intensive Health Care Unit", David Bracewell, et al.(Eds):AIAA 2011, CS \& IT 03, pp.169-176,2012

[6] S.Joephine Selvarani, "Online Health Monitoring System", International Journal on computer science and engineering, Vol.3 no. 4 April 2014.

[7] Resource Aware Mobile Based Health Monitoring, Mohammad M. Masud, Mohamed Adel Serhani, and AlramzanaNujumNavaz. LINDSEY S RAGHAVENDRA C S. PEGASIS,

[8] "Power- Efficient Gathering in Sensor Information Systems," Proceedings of the IEEE Aerospace Conference, pp.1125-1130, 2002.

[9] Manjeshwar A, Agrawal D. Teen, "A Routing Protocol for EnhancedEfficiency in Wireless Sensor Networks," Proceedings of the 15thParallel and Distributed Processing Symposium, pp.2009-2015, 2001.

[10] Enrique Dorronzoro Zubiete Luis Fernandez Luque Ana Veronica Medina Rodr'iguez, Isabel G'omez Gonz'alez " Review of wireless sensors networks in health applications" 33rd Annual International

[11] Conference of the IEEE EMBS Boston, Massachusetts USA, August 30September 3, 2014. [5] R. Prakash1,B.Paulchamy2 "Remote Monitoring Of ECG And Body Temperature Signals" ISSN: 2319-8753, May 2014.

[12]M.Abo-Zahhad, Sabah M. Ahmed and O.Einahas,"A wireless Emergency Telemedicine system for patients monitoring and Diagnosis"IJTA,vol.2014,Jan.2014.

[13]V.G.Girhepunje, Santosh Chede, "Design and Implementation of Wearable Wireless Health Monitoring Module", Journal of Applied Science and Computationas (JASC), Volume 5, Issue 5, May2018,ISSN No. 0076-5131, Pp 78-83.

[14] Texas Instruments, User guide on" MSP-EXP432P401R Launch Pad ${ }^{\mathrm{TM}}$ Evaluation Kit"

[15]Texas instruments's an 'application report' and 
"MSP432"M Platform Porting Guide "2015

[16]MSP432 ${ }^{\mathrm{TM}}$ microcontrollers: Bringing high performance to low- power applications data sheet,2015

[17]DS18B20 Programmable Resolution -1 wire digital thermometer-

[18] Overview by Maim Integrated,2012

[19] Analog devices,single lead heart rate monitor front end AD 8232 data sheet 2015

[20]M.S.Balamurugan, A/P, ECE, M.P.Ajay, A/P, ECE, Cloud Care: A Remote Health Monitoring System, IWBBIO 2013. Proceedings Granada, 18-20 March 2013 IEEE.

[21]C.P. Figueiredo, Student Member, IEEE, K. Becher, Member, IEEE, K.-P. Hoffmann, Member, IEEE, P.M. Mendes, Member, IEEE. "Low Power Wireless Acquisition Module for Wearable Health Monitoring Systems." 978-1-4244-4124-2/10/\$25.00 @2010 IEEE

[22]Rashkovska, I. Tomašić and R. Trobec, "A Telemedicine Application: ECG Data from Wireless Body Sensors on a Smartphone” MIPRO 2011, May 23-27, 2011,Opatija Croatia.

[23] Shenzhen Anxinke Technology company ltd."ESP8266/12-E data sheet.

[24]Basant Kumar, S. P. Singh* and Anand Mohan* ", Emerging Mobile Communication Technologies for Health" int'l conf.on computer and communication technology ICCCT'10, 978-1-4244- 9034/10/\$26.00@2010 IEEE. 